\title{
Oligopeptidase B from Leishmania amazonensis: molecular cloning, gene expression analysis and molecular model
}

\author{
Herbert Leonel de Matos Guedes • \\ Monique Pacheco Duarte Carneiro • \\ Daniel Cláudio de Oliveira Gomes • \\ Bartira Rossi-Bergmann • \\ Salvatore Giovanni De-Simone
}

Published online: 17 July 2007

(C) Springer-Verlag 2007

\begin{abstract}
Serine oligopeptidases of trypanosomatids are emerging as important virulence factors and therapeutic targets in trypanosome infections. A complete open reading frame of oligopeptidase B from Leishmania amazonensis was amplified with polymerase chain reaction with gradient annealing temperatures using primers designed for the oligopeptidase B gene from L. major. The 2,196-bp
\end{abstract}

The name of Dr Bartira Rossi-Bergmann is spelled incorrectly in the article. Please see the correct spelling above.

The online version of the original article can be found at http://dx.doi. org/10.1007/s00436-007-0552-5.

H. L. de Matos Guedes • M. P. D. Carneiro - S. G. De-Simone

Laboratório de Bioquímica de Proteínas e Peptídeos,

Departamento de Bioquímica e Biologia Molecular,

Fundação Oswaldo Cruz,

Rio de Janeiro, RJ, Brazil

H. L. de Matos Guedes • D. C. de Oliveira Gomes •

B. Rossi-Bergmann

Laboratório de Imunofarmacologia, Instituto de Biofísica Carlos

Chagas Filho, Universidade Federal do Rio de Janeiro,

Rio de Janeiro, RJ, Brazil

S. G. De-Simone

Departamento de Biologia Celular e Molecular,

Instituto de Biologia, Universidade Federal Fluminense,

Niterói, RJ, Brazil

S. G. De-Simone $(\bowtie)$

Fundação Oswaldo Cruz,

Av, Brasil, 4365. Pavilhão 26-Sala 309,

CEP: 21045-900 Rio de Janeiro, RJ, Brazil

e-mail: dsimone@ioc.fiocruz.br fragment coded for a protein of 731 amino acids with a predicted molecular mass of $83.49 \mathrm{KDa}$. The encoded protein (La_OpB) shares a 90\% identity with oligopeptidases of $L$. major and $L$. infantum, $84 \%$ with $L$. braziliensis, and $\sim 62 \%$ identity with Trypanosoma peptidases. The oligopeptidase B gene is expressed in all cycle stages of L. amazonensis. The three dimensional model of La_OpB was obtained by homology modeling based on the structure of prolyl oligopeptidases. We mapped a La_OpB model that presents a greater negative charge than prolyl oligopeptidases; our results suggest a difference in the S2 subsite when compared to oligopeptidases B from Trypanosoma and bacterial oligopeptidases B. The La_OpB model serves as a starting point for its exploration as a potential target source for a rational chemotherapy.

\section{Introduction}

The leishmaniases are parasitic diseases affecting millions of people worldwide, with a wide range of clinical symptoms caused by the protozoa of the genus Leishmania (Murray et al. 2005). Parasites of the genus Leishmania present two forms in their life cycle: the spindle-shaped flagellated promastigotes, which multiply in the midgut of the sandfly vector and oval non-motile amastigotes, obligate intracellular forms that live inside the vertebrate hosts tegument and visceral tissue mononuclear phagocyte system cells. Depending on the species, Leishmania causes a spectrum of diseases ranging from self-healing cutaneous lesions to lethal visceral consequences (Alexander and Russell 1992).

American cutaneous leishmaniasis is a disease clinically characterized by ulcerated skin lesions at the site of the 
insect bite that often heal spontaneously (Coutinho et al. 1987). A small percentage of affected individuals develop diffuse cutaneous leishmaniasis after the healing of the primary lesion, which is clinically characterized by a diffuse infiltration of the skin on which appear a large number of nodules, papules, tubercules, and infiltrated plaques that rarely become ulcerated (Ready et al. 1983). In older cases of the disease, disseminated lesions may cover much of the body, but are predominantly on the extremities and rarely involve the nasopharyngeal mucous membranes (Barral et al. 1995). These patients suffer from an immunological incapacity to develop antigen-specific cellmediated reactivity to Leishmania. L. amazonensis is the main agent of the anergic diffuse cutaneous leishmaniasis.

Diffuse cutaneous leishmaniasis presents a very difficult therapeutic problem and frequent failure to respond to conventional antimony treatment. Furthermore, the drugs of choice are significantly toxic and expensive, and individuals frequently suffer refractory infections. Thus, the identification and research into new drug targets is necessary, and the development of novel, more effective, and less toxic drugs is an urgent priority (Croft and Coombs 2003).

Protozoan proteases play crucial roles in the hostparasite interaction, and their characterization contributes to the understanding of the protozoan disease mechanisms. Proteases are important for parasite survival; they are involved in the digestion of exogenous proteins for nutritive purposes (Rosenthal 1999), invasion of host cells and tissues (Roggwiller et al. 1996), and modification of host proteins (Caler et al. 1998). The proteases have been considered as preeminent targets group in several pathologies, and the importance of Leishmania proteases has been confirmed by the finding that specific protease inhibitors kill parasites and reduce the evolution of leishmaniatic lesions (Sadij and McKerrow 2002).

Oligopeptidase B is a serine oligopeptidase of the prolyl oligopeptidase family (clan SC, family S9; Rawlings et al. 2006; Venäläinen et al. 2004). This enzyme family is different from the classical serine protease families, trypsin, and subtilisin in that the enzymes cleave only peptide substrates while excluding large proteins (Polgar 2002). The enzyme consists of a peptidase domain with an $a / b$ hydrolase fold and seven-bladed $\beta$-propeller domains (Gerczei et al. 2000), similar to the prolyl oligopeptidase (Rea and Fulop 2006; Fülöp et al. 1998). Oligopeptidases B cleaves peptides at lysine and arginine residues (Pacaud and Richaud 1975; Polgar 2002), although they have an enhanced catalytic efficiency for dibasic substrates (Ashall et al. 1990; Polgar 1997; Morty et al. 2002; Hemerly et al. 2003).

Trypanosomatid oligopeptidase B is emerging as an important virulence factor (Burleigh and Woolsey 2002) and therapeutic target in trypanosome infections (Cazzulo 2002). A major signaling pathway regulating cell invasion by Trypanosoma cruzi involves mobilization of $\mathrm{Ca}^{+2}$ from intracellular stores and requires the activity of oligopeptidase B (Burleigh et al. 1997). Oligopeptidase B from $T$. evansi inactivated atrial natriuretic factor in the bloodstream of the infected host (Morty et al. 2005). Furthermore, enzymes from African trypanosomatids are active in the blood stream of infected hosts (Morty et al. 2001), and the drugs most commonly used in sleeping sickness treatment reduce the activity of these serine oligopeptidases (Morty et al. 1998).

Previous studies in the Leishmania ssp demonstrate the presence of serine proteases (Colmenares et al. 2001; Silva-Lopez and De-Simone 2004a, b; Silva-Lopez et al. 2005; Guedes et al. 2007) and serine oligopeptidases (Ribeiro de Andrade et al. 1998). Oligopeptidase B of $L$. major was first demonstrated by Morty et al. (1999) and then annotated again by the genome project (Ivens et al. 2005), confirming the presence of this enzyme that has already been characterized in other trypanosomatids. In this study, primers designed from the L. major oligopeptidase B gene were used to clone a complete coding sequence of oligopeptidase from $L$. amazonenis. Comparison of the deduced amino acid sequences of oligopeptidase B from $L$. amazonensis demonstrate that Leishmania oligopeptidases $\mathrm{B}$ are closely related in terms of sequence identity and subsites and demonstrate differences against oligopeptidases B from Trypanosoma species.

\section{Materials and methods}

Parasites

L. amazonensis (IFLA/BR/67/PH8), L. major (MRHO/SU/ 59/P), L. chagasi (MHOM/Br/1974/PP75), and L. braziliensis (MHOM/BR/1975/M029) promastigote forms were maintained at $26^{\circ} \mathrm{C}$ in M199 with $10 \%(v / v)$ heatinactivated fetal-calf serum. Axenic amastigotes from $L$. amazonensis was obtained by in vitro promastigoteamastigote differentiation. Briefly, promastigotes from the late $\log$ phase of growth $\left(10^{7}\right.$ cells $\left./ \mathrm{ml}\right)$ were washed three times in phosphate buffer saline, $\mathrm{pH}$ 7.2, PBS $\left(3,000 \times g, 15 \mathrm{~min}, 5^{\circ} \mathrm{C}\right)$ and incubated in Schneider's Drosophila medium with $20 \% \mathrm{FCS}$, pH 5.5 , at $34^{\circ} \mathrm{C}$ for $96 \mathrm{~h}$ (Alves et al. 2005). Cell numbers were estimated by counting the parasites in a Newbauer chamber.

\section{PCR assay}

Genomic DNA was extracted from $1 \times 10^{8}$ promastigotes according to Sambrook and Russell (2001). DNA quanti- 
fication was performed in a Gene Quant pro RNA/DNA calculator. The polymerase chain reaction (PCR) was performed with $100 \mathrm{ng}$ of extracted DNA using a OLIB_F1 and OLIB_R1 primer set designed based on the nucleotide sequence of oligopeptidase B fom L. major (Table 1). The PCR amplified in a $25 \mu \mathrm{l}$ of reaction volume containing $1 \mathrm{U}$ of Platinum Taq (Invitrogen), $1 \times$ Platinum Taq buffer, $1.5 \mathrm{mM} \mathrm{MgCl} 2,200 \mu \mathrm{M}$ of deoxynucleosides triphosphates, and $20 \mathrm{pMol}$ of each primer. Two different reactions were performed. For $L$. major, the amplification program was carried out as follows: $95^{\circ} \mathrm{C}$ for $5 \mathrm{~min}, 35$ repeated cycles of $1 \mathrm{~min}$ at $95^{\circ} \mathrm{C}, 1 \mathrm{~min}$ at $68^{\circ} \mathrm{C}$, and $2 \mathrm{~min}$ at $72^{\circ} \mathrm{C}$, followed by a single terminal extension at $72^{\circ} \mathrm{C}$ for $15 \mathrm{~min}$. For L. amazonensis, the amplification program was carried out as follows: $95^{\circ} \mathrm{C}$ for $5 \mathrm{~min}$, ten cycles of $1 \mathrm{~min}$ at $95^{\circ} \mathrm{C}$, $1 \mathrm{~min}$ at $68^{\circ} \mathrm{C}$, and $2 \mathrm{~min}$ at $72^{\circ} \mathrm{C}$; this cycle was repeated ten times with different primer annealing temperatures at $64,60,55,50,45$ and $64^{\circ} \mathrm{C}$ followed by a single terminal extension at $72^{\circ} \mathrm{C}$ for $15 \mathrm{~min}$. All reactions were performed in Eppendorf Matercycler.

\section{Cloning and sequencing}

The PCR products were electrophoresed in $1 \%$ agarose (Sigma). A single band spanning the entire L. major and $L$. amazonensis open reading frames (ORFs) was purified and cloned in the pGem $\mathrm{T}$ cloning vector. The plasmids were sequenced automatically using specific primers and primers from the vector (Table 1).

Detection of products expressed from the $L$. amazonensis oligopeptidase B Gene

Total RNA was extracted from approximately $1 \times 10^{8}$ promastigote cells in the logarithmic growth phase and stationary phase (5 days of culture) of culture and axenic amastigotes by homogenization in $1 \mathrm{ml}$ of Trizol reagent (Invitrogen). DNA contamination was eliminated by treat-

Table 1 Primer sets used for PCR amplification and sequencing of the putative oligopeptidase B from L. amazonensis

\begin{tabular}{ll}
\hline Primers & Sequence \\
\hline OLIB-F1 $^{\text {a }}$ & atg tcg tcg gac agc tcc gtc gcg \\
OLIB-R1 $^{\text {a }}$ & cct gcg aac cag cag gcg cac ggt \\
OLIB-F2 $^{\text {b }}$ & gag gac gtc tgc ctc tac gag gag \\
OLIB-R2 & ctc act gcc gcc gcg gat gtg ggc \\
M13-F & cgc cag ggt tt ccc agt cac gac \\
M13-R & tca cac agg aaa cag cat tgac
\end{tabular}

${ }^{\text {a }}$ Oligonucleotides designed based on the L. major sequence found in the database

${ }^{\mathrm{b}}$ Oligonucleotides designed from internal fragments obtained from the L. amazonensis oligopeptidase B sequence

${ }^{\mathrm{c}}$ Oligonucleotides from pGEM $\mathrm{T}$ cloning vector (Promega)
Table 2 Amino acid sequence alignments of oligopeptidase B from $L$. amazonensis using Blast 2 sequences

\begin{tabular}{ll}
\hline & Amino acid (\%) \\
\hline Lm Q4QHU7 & $90(95)$ \\
Li-genome & $90(95)$ \\
Lb-genome & $84(93)$ \\
Tc Q94795 & $63(77)$ \\
Tb O76728 & $62(77)$ \\
Te Q6QDP1 & $62(76)$ \\
Ec P24555 & $37(53)$ \\
Se Q9L6C8 & $37(54)$
\end{tabular}

ment with Dnase I (Boehringer Mannheim, Germany). RNA was quantitated using a spectrophotometer, and $5 \mu \mathrm{g}$ of RNA was used to synthesize cDNA with the SuperScript Indirect cDNA labeling kit (Invitrogen). The expression level of oligopeptidase B gene was estimated by PCR using specific primers (OliB-F2 and OliB-R2). Primers designed for the ITS region were used as controls (Dávila 2002).

Sequence analysis

Sequences of oligopeptidases B were extracted from swissprot/TrEMBL and from the genome project of $L$. braziliensis (MHOM/BR/75M2904) and L. infantum clone JPCM5 (MCAN/ES/98/LLM-877). Molecular mass and pI were determined by the protParam program (Gasteiger et al. 2005). The scan-prosite was used to find the prolyloligopeptidase signature (de Castro et al. 2006). The presence of putative signal peptides was evaluated by the prediction of signal peptide cleavage sites using SignalIP (Bendtsen et al. 2004), and the prediction of mitochondrial targeting sequences was performed using MitoProt (Claros and Vincens 1996). Sequence alignments were carried out with protein-protein basic local alignment search tool BLAST 2 sequences using default parameters (Altschul et al. 1997) and with ClustalW using default parameters (Higgins et al. 1994). All programs are located at http:// www.expasy.org.

Molecular modeling

Prolyl oligopeptidase from porcine muscle (PDB ID 1QFM A, 1.40-Å resolution; Fülöp et al. 1998) and Myxococcus xanthus (PDB ID 2BKL 1.5- $\AA$ resolution; Shan et al. 2005) were used as templates in the modeling procedure. A reliable alignment between $\mathrm{La} \mathrm{OpB}$ and their templates was achieved by adjusting the sequence alignment obtained from ClustalW v. 1.72 (Higgins et al. 1994). The alignment of N-terminal (46-448) with prolyl oligopeptidase from porcine muscle was submitted to the Swiss-model alignment interface of the Swiss-model automated modeling 
Fig. 1 PCR of oligopeptidase B. a PCR of genomic DNA from $L$. major using annealing temperature at $69^{\circ} \mathrm{C}$. b PCR gradient of genomic DNA from L. amazonensis (lane 1), L. braziliensis (lane 2), and $L$. chagasi (lane 3). c PCR gradient of genomic DNA from L. amazonensis in the absence (lane 1) and in the presence (lane 2) of dimethyl sulfoxide a

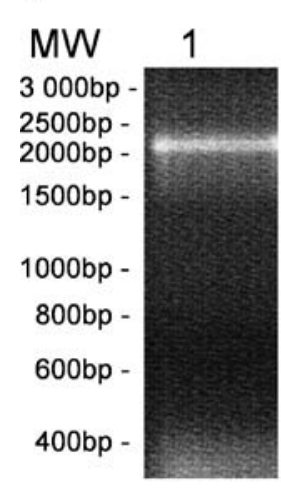

b

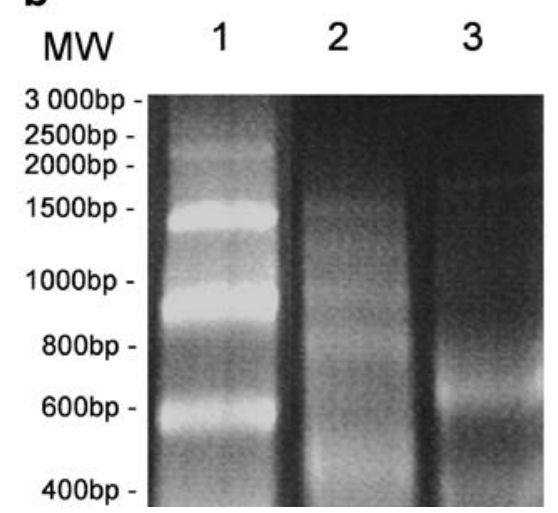

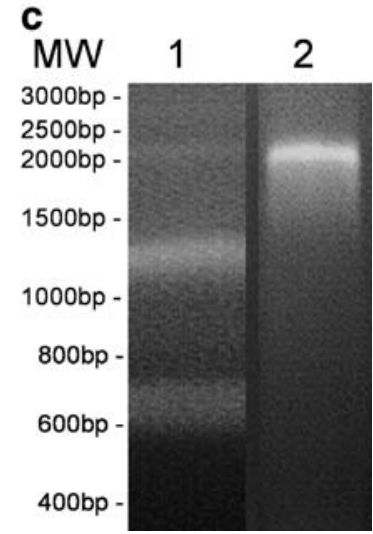

server (Guex and Peitsch 1997) to derive a 3D model for the $\mathrm{N}$ terminal. The alignment of the $\mathrm{C}$ terminal (449-731) with prolyl oligopeptidase from porcine muscle and $M$. xanthus was submitted to optimize the model approach with the Swiss-model automated modeling server. Both structures were then merged using the SwissPDB viewer v. 3.6 program, and the suitability of the modeled La_OpB three dimensional (3D) structure for this study was assessed with the analytical tools available in SwissPDB viewer v. 3.6 and with the programs in the PROCHECK suite for model validation (Laskowski et al. 1993). The molecular model of La_OpB was submitted to energy minimization for model refinement, a procedure similar to that used in the molecular modeling of Lachesis muta muta thrombin-like enzyme (Silva and De-Simone 2004). Energy minimization operations described in this section were conducted with the GROMOS96 v.43B1 force field implemented in the SwissPDB viewer v.3.7b2. La_OpB were minimized using a harmonic constraint with 20 steps of steepest descent followed by 1,000 steps of steepest descent and conjugated gradient minimization methods until the energy difference between the two steps was below $0.01 \mathrm{~kJ} / \mathrm{mol}$. Two successive refinements of the La_OpB model were performed using a similar minimization protocol in which the type of residues constrained during the minimization were varied: Initially, only residues out of the most favored regions of the Ramachandran plot were allowed to move; then,

Table 3 Scan prosite analysis of oligopeptidase from Leishmania ssp

\begin{tabular}{lll}
\hline & $\begin{array}{l}\text { Amino acid } \\
\text { position }\end{array}$ & $\begin{array}{l}\text { Prolyl endopeptidase family serine active site } \\
(\text { PATTERN) (PS00708) }\end{array}$ \\
\hline $\mathrm{La}$ & $552-582$ & DfiaAaefLvdaklttpsqlaceGrSaGGLL \\
$\mathrm{Lm}$ & $552-582$ & DfiaAaefLvnaklttpsqlaceGrSaGGLL \\
$\mathrm{Li}$ & $552-582$ & DfiaAaefLvnaklttpsqlaceGrSaGGLL \\
$\mathrm{Lb}$ & $552-582$ & DfiaAaecLvdakmttpsqlaceGrSaGGLL
\end{tabular}

${ }^{a}$ Consensus pattern according to prosite analysis of the prolyl endopeptidase family serine active site: $\mathrm{D}-\mathrm{x}(3)-\mathrm{A}-\mathrm{x}(3)-[\mathrm{LIVMFYW]}-$ x(14)-G-x-S-x-G-G-[LIVMFYW](2)

$S$ Active site residue residues with a high model B-factor and/or force field energy were included. The final refined theoretical structure of La_OpB was achieved by an energy minimization with 20 steps of steepest descent in all residues. Refinement progress was accompanied by the distribution of La_OpB residues over the most favored and allowed regions of the Ramachandran plot. The molecular surfaces were calculated in SwissPDB viewer v. 1.6 (Guex and Peitsch 1997) and colored according to the coulombic electrostatic potential: red-negative, blue-positive, and white-neutral. We compared a molecular model of La_OpB against prolyl oligopeptidase from $M$. xanthus (PEP_Mx). All procedures were performed on a Pentium IV personal computer.

\section{Results and discussion}

Cloning, sequencing, and analysis of the oligopeptidase B gene

The oligopeptidase gene isolated from L. amazonensis consisted of an ORF of 2,193 bp (Fig. 1) encoding a polypeptide of 731 amino acids (Fig. 3) with a predicted molecular mass of $83.52 \mathrm{kDa}$ and a predicted $\mathrm{p} I$ value of 5.61. This sequence was submitted to GenBank with accession number EF392367. The encoded polypeptide

Table 4 Mapping of trypanosomatid oligopeptidase B catalytic domains and subsites by homology with bacterial oligopeptidases B

\begin{tabular}{llllllll}
\hline & Ser & Asp & His & S1 & S1 & S2 & S2 \\
\hline E. coli & 532 & 617 & 652 & E576 & E578 & D460 & D462 \\
Se & 532 & 617 & 652 & E576 & E578 & D460 & D462 \\
La & 577 & 662 & 697 & E621 & E623 & D504 & Q506 \\
Lc & 577 & 662 & 697 & E621 & E623 & D504 & Q506 \\
Lb & 577 & 662 & 697 & E621 & E623 & D504 & K506 \\
Lm & 577 & 662 & 697 & E621 & E623 & D504 & Q506 \\
Tc & 562 & 647 & 682 & E606 & E609 & E489 & E491 \\
$\mathrm{Tb}$ & 563 & 648 & 683 & E607 & E609 & E490 & E492 \\
Te & 563 & 648 & 683 & E607 & E609 & E490 & E492 \\
\hline
\end{tabular}




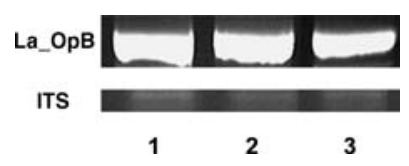

Fig. 2 RT-PCR of oligopeptidase B. a Oligopeptidase B genes were amplified by PCR in cDNA from promastigotes in the stationary phase (lane 1), promastigotes in the logarithmic growth phase (lane 2), and axenic amastigotes from L. amazonensis (lane 3). ITS primers were used as controls for cDNA. No reactions were observed in the samples of the control without reverse transcriptase (results not shown)

chain contained a prolyl endopeptidase family serine active site signature sequence (Table 3). The encoded oligopeptidase B from Leishmania shared $90 \%$ identity to L. major and L. infantum, $84 \%$ to L. braziliensis, $\sim 67 \%$ to trypanosomes oligopeptidases, and $37 \%$ to bacterial oligopeptidases (Table 2). The identity observed between Leishmania oligopeptidases and trypanosomes oligopeptidases is similar to that observed between oligopeptidases from T. cruzi and T. brucei ( 71\%). Sequence alignments with oligopeptidases B of trypanosomatids indicated conservation in the entire sequence; therefore, conservation of bacterial oligopeptidases appears to be located in the carboxy-terminal. In the prolyl oligopeptidase family, the amino acid sequence homology is more significant in the carboxyl terminal domain than in the amino terminal due to the fact that the catalytic domain is concentrated in the carboxyl terminal region within about 200 residues. Two large insertions were observed in trypanosomatids at position 34 to 44 (GPN PMNPPRHH) and at position 381 to 388 (QDGVFKPG) of L. amazonensis in relation to bacterial oligopeptidases B. Leishmania oligopeptidases B display an $\mathrm{N}$ terminal extension at position 1 to 13 (MSSDSSVAASVQP) and one insertion at position 189 to 193 (SDPCR) that is absent in trypanosome oligopeptidases B. Oligopeptidase B has been characterized as a cytosolic protease (Polgar 2002). We did not observe any putative signal peptide at the $\mathrm{N}$ terminal extension using SignalIP and MitoProt; this finding is in agreement with the hypothesis that oligopeptidases B do not have a zymogen (or proenzyme) form and are apparently synthesized as active peptidases.

The oligopeptidase B gene is present as a single copy in Leishmania ssp genomes and expressed during all life cycle stages of $L$. amazonensis

In silico bioinformatic, analysis of all Leishmanias genomes suggests that oligopeptidase B is a single copy gene; however, genomes were not conclusive/included. The oligopeptidase B gene is located at chromosome 9 in $L$. major, L. braziliensis, and L. infantum. In T. cruzi and T. brucei, Southern blot analysis characterized oligopeptidase B gene as a single copy. atgtcgtcggacagctccgtcgcggcctctgtgcagccgccgatcgccgccaagaagccg

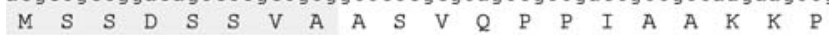
caccgegtcacgttcggetacgtggagggtgaggaccgcggeccgaacccgatgaacccg

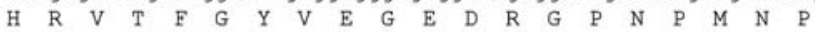
ccgcgccatcacgaggacccatacttttggatgcgggacgacgatcgcaaggatccggec

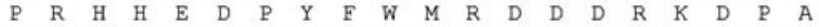
gtgattgaacacctcaaaaaggagaaggcctact tcgaggcgtgcagctccgacatgacC $\begin{array}{llllllllllllllllllll}V & I & E & H & L & K & K & E & K & A & Y & F & E & A & C & S & S & D & M & T\end{array}$ cagctgcgcgacgacatctacacggagcacatttcgcacataaaggaagatgacatgtct $\begin{array}{llllllllllllllllllll}Q & L & R & D & D & I & Y & T & E & H & I & S & H & I & K & E & D & D & M & S\end{array}$ gcgccgtacctgtacggccagtaccggtactacacccgcgaggtgaagggtaagtcgtac $\begin{array}{llllllllllllllllllllllllllllllll}A & P & Y & L & Y & G & Q & Y & R & Y & Y & T & R & E & V & K & G & K & S & Y\end{array}$ aagatttactgcegcgtgcccaaggacaaggagecgggggacgtcgeggtcgaggaggtc

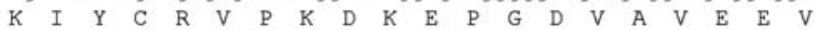
gtcatcgatgtcaacaaggtcgccgagggcaaaccgttctgtgacgtgatggaggtggag

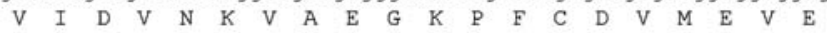
ccggcaccgccggagcacgacctcgtggccttctctgtggacatgagcggtaacgaggtg

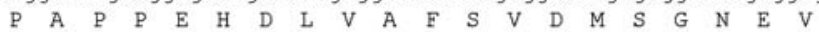
tacgcgatcgaatttaagcatatgtcggacccgtgccggactgtagcggacacggtgagc $\begin{array}{llllllllllllllllllll}Y & A & I & E & F & K & H & M & S & D & P & C & R & T & V & A & D & T & V & S\end{array}$ ggcactaatggcgagatcgtgtggggcccggaccacacctccttttctacgtgacgaag $\begin{array}{llllllllllllllllllllllllll}G & T & N & G & E & I & V & W & G & P & D & H & T & S & F & F & Y & V & T & K\end{array}$ gacgaaacgctgcgcgaaaacaaagtgtggcgccacgtgatgggcaggccgcagtccgag $\begin{array}{lllllllllllllllllllll}D & E & T & L & R & E & N & K & V & W & R & H & V & M & G & R & P & Q & S & E\end{array}$ gacgtctgcetctacgaggagaacaacccgctgttcagcgccttcatgtacaaggccgcC

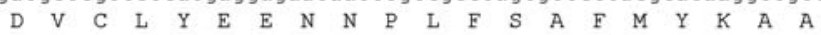
gacacaaacacctttgcatcggctcgcagtcgtcggagacgacagaggtgcacctgctt $\begin{array}{llllllllllllllllllll}D & T & N & T & L & C & I & G & S & Q & S & S & E & T & T & E & V & H & L & L\end{array}$ gatctgcgccagggcaacgcacacaataccgttgaagttgtgcggccgcgcgagaagggt $\begin{array}{lllllllllllllllllllll}D & L & R & Q & G & N & A & H & N & T & V & E & V & V & R & P & R & E & K & G\end{array}$ gtgcgetacaacgtacagatgcacggcacccgccatcttctgatcctcaccaacaaagac

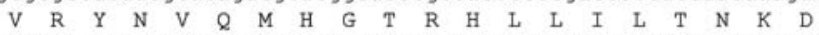
ggggeggtgaaccacaagcttctcatagcgecgegegggcagccgagcgactggtcacat

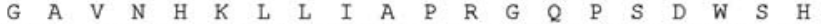
gtgctggtggatcacaccgaggacgtgtttatggagaacatcgcggtgcgetcgaactac

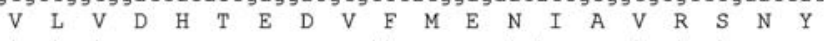
ctcgtcgtgacaggccgccgcggcgggttgacgcgcatctggacgatgatggtggacccg

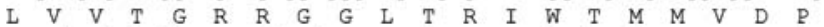
caggatggtgtcttcaagcctggtgccgagctgcgcgaggtgatgatggaggaaccgatc

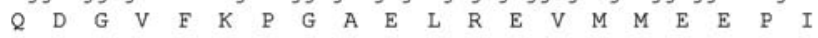
ttcacggtgcacctcgtggagtcccagatgttggagtacgaagagtcgacattccgcatg

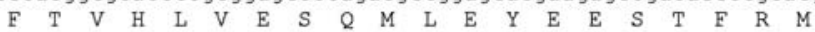
gagtactcgtcccttgccacgccgaacacgtggttcaacgtcagcccgcaggaccactct

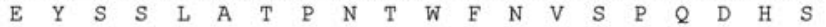
cgcaccgttgtaaaagtgcgcgaggtcggcggtggcttcgacgccgccaactacaaggtg

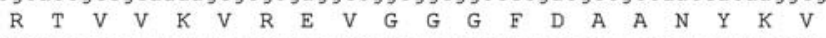
gagcgccgcttcgctaccgcaccggaccagaccaagatcccgctttcacttgtctaccac

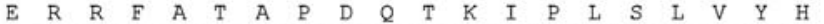
aaagacctcgacatgacccagccgcagccgtgcatgctgtacgggtacggcagctacggc

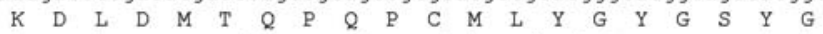
atcagcatggaccccagttcaccattcagcatctgccgtactgtgaccgcggcatgatc

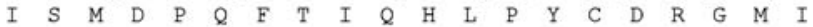
tacgtcatagcccacatccgcggcggcagtgagatgggccgtgcatggtacgagatcggc $\begin{array}{lllllllllllllllllllll}Y & V & I & A & H & I & R & G & G & S & E & M & G & R & A & W & Y & E & I & G\end{array}$ gccaagtacctcacgaagcgcaacaccttttcggacttcatcgcggcagccgagttcctg

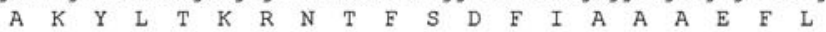
gtggatgcgaaattgacgacgccgtcgcagctggcctgcgaggggcgtagcgccggcggC $\begin{array}{llllllllllllllllllll}V & D & A & K & L & T & T & P & S & Q & L & A & C & E & G & R & S & A & G & G\end{array}$ ctgctggtgggcgcggtgctgaacatgcgtcctgatctcttcaaggtggcgctcgccggc

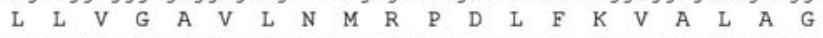
gtgccgttcgtggatgtcatgacgaccatgtgcgacccagcattccettgacgacgggc

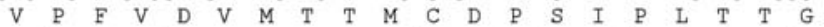
gagtgggaggagtggggaaacccgaacgagtacaagtactacgactacatgctgagctac

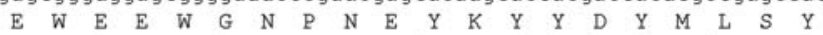
agccceatggacaacgtccgcgcgcaggagtacccgaatatcatggtccagtgcggectg

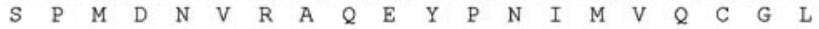
cacgaccccgcgtcgcctattgggaaccggccaagtgggtgagcaagctgcgtgagcac

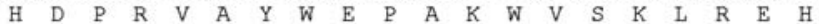
aagacagactgcaacgaa attctgctgaacatggacatggagagcggacacttctccgcC

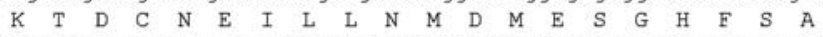
agggatcgctacaagttttggaaggagtcggctgtccagcaagcgttcgtgtgcaagcac $\begin{array}{llllllllllllllllllll}R & D & R & Y & K & F & W & K & E & S & A & V & Q & Q & A & F & V & C & K & H\end{array}$ ctgaagagcaccgtgcgcctgctggttcgcagg

$\begin{array}{llllllllllll}\text { L } & K & S & T & V & R & L & L & V & R & R\end{array}$

Fig. 3 Nucleotide and deduced amino acid sequence of oligopeptidase B from L. amazonensis. The amino acid sequence is shown immediately below the nucleotide sequence. We demonstrated the primer region highlighted in gray. This sequence was submitted to GenBank with accession number EF392367 
Fig. 4 Multiple alignment of the $\mathrm{N}$ terminal of oligopeptidase B sequences. The amino acid sequences of $L$. amazonenis (this work), L. major (accession number Q4QHU7), L. infantum (Genome project: LinJ09.0820). L. braziliensis (Genome project: LbrM09_V2.0850), T. cruzi (accession number Q94795), $T$. brucei (accession number O76728), T. evansi (accession number Q6QDP1), E. coli (accession number P24555), and $S$. entérica (accession number Q9L6C8) were aligned with ClustalW at EBI (expasy proteomics tools). The catalytic triad was highlighted in dark gray. S1 and S2 subsites were highlighted in gray. The asterisks indicate the positions of highly conserved amino acid residues. The alignment was performed at Clustal W
Tb DTSIDLKKPNPTMLYGYGSYGICIEPEFNSRFLPYVDRGMIYAIAHVRGGGEMGRTWYEV 525 Te DTSIDLKKPNPTMLYGYGSYGICIEPEFNSRFLPYVDRGMIYAIAHVRGGGEMGRTWYEV 525 TC DVSLDMSKPHPTVLYAYGSYGACVEPEFSVKYLPYLDRGVIYVIAHVRGGGEMGRAWYEV 524 LC HKDLDMSQPQPCMLYGYGSYGLSMDPQFSIQHLPYCDRGMIFAIAHIRGGSEMGRAWYEI 539 Lm HKDLDMSQPQPCMLYGYGSYGLSMDPQFSIQHLPYCDRGMIFAIAHIRGGSELGRAWYEI 539 La HKDLDMTQPQPCMLYGYGSYGISMDPQFTIQHLPYCDRGMIYVIAHIRGGSEMGRAWYEI 539 Lb HKDLDVSQPQPCMLYGYGSYGLCVDPKFSIQHLPYCDRGMIYAIAHIRGGSEMGRAWYEI 539 EC HRKHFRKGHNPLLVYGYGSYGASIDADFSFSRLSLLDRGFVYAIVHVRGGGELGQQWYED 495 Se HQKYFRKGQNPLLVYGYGSYGSSIDADFSSSRLSLLDRGFVYAIVHVRGGGELGQQWYED 495

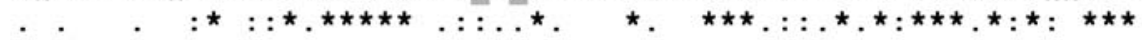

Tb GGKYLTKRNTFMDFIACAEHLISSGLTTPAQLSCEGRSAGGLLVGAVLNMRPDLFHVALA 585 Te GGKYLTKRNTFMDFIACAEHLISSGLTTPAQLSCEGRSAGGLLVGAVLNMRPDLFHVALA 585 TC GAKYLTKRNTFSDFIACAEYLIEIGLTTPSQLACEGRSAGGLLIGAVLNMRPDLFRVALA 584 LC GAKYLTKRNTFSDFIAAAEFLVNAKLTTPSQLACEGRSAGGLLVGAVLNMRPDLFKVALA 599 Lm GAKYLTKRNTFSDFIAAAEFLVNAKLTTPSQLACEGRSAGGLLMGAVLNMRPDLFKVALA 599 La GAKYLTKRNTFSDFIAAAEFLVDAKLTTPSQLACEGRSAGGLLVGAVLNMRPDLFKVALA 599 Lb GAKYLTKRNTFSDFIAAAECLVDAKMTTPSQLACEGRSAGGLLVGTVLNMRPDLFKAALA 599 EC G-KFLKKKNTFNDYLDACDALLKLGYGSPSLCYAMGGSAGGMLMGVAINQRPELFHGVIA 554 Se G-KFLKKRNTFNDYLDACDALLKLGYGSPSLCYGMGGSAGGMLMGVAINERPELFHGVIA 554

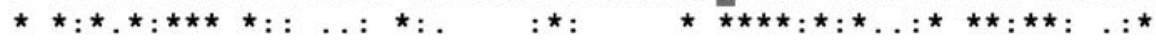

Tb GVPFVDVMTTMCDPSIPLTTGEWEEWGNPNEYKFFDYMNSYSPIDNVRAQDYPHLMIQAG 645 Te GVPFVDVMTTMCDPSIPLTTGEWEEWGNPNEYKFFDYMNSYSPIDNARAQDYPHLMIQAG 645 TC GVPFVDVMTTMCDPSIPLTTGEWEEWGNPNEYKFFDYMNSYSPVDNVRAQDYPHLMIQAG 644 LC GVPFVDVMTTMCDPSIPLTTGEWEEWGNPNEYKYYDYMLSYSPVDNVRAQEYPNIMVQCG 659 Lm GVPFVDVMTTMCDPSIPLTTGEWEEWGNPNEYKYYDYMLSYSPMDNVRAQEYPNIMVQCG 659 La GVPFVDVMTTMCDPSIPLTTGEWEEWGNPNEYKYYDYMLSYSPMDNVRAQEYPNIMVQCG 659 Lb GVPFVDVMTTMCDPSIPLTTGEWEEWGNPNEYKYYDYMLSYSPVDNVRAQAYPNIMIQSG 659 EC QVPFVDVVTTMLDESIPLTTGEFEEWGNPQDPQYYEYMKSYSPYDNVTAQAYPHLLVTTG 614 Se QVPFVDVLTTMLDESIPLTTGEFEEWGNPQDIEYYDYMKSYSPYDNVKAQDYPHLLVTTG 614

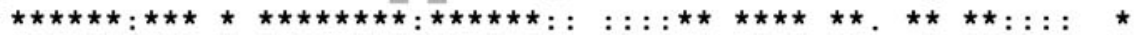

Tb LHDPRVAYWE PAKWASKLRELKTDSNEVLLKMDLESGHFSASDRYKYLRENAIQQAFVLK 705 Te LHDPRVAYWE PAKWASKLRELKTDSNEVLLKMDLESGHFSASDRYKYLRENAIQQAFVLK 705 TC LHDPRVAYWE PAKWASKLRALKTDSNEVLLKMDLESGHFSPSDRYRYWREMSFPQAFVLK 704 LC LHDPRVAYWE PAKWVSKLRECKTDNNE ILLNMDMESGHFSAKDRYKFWKESAIQQAFVCK 719 Lm LHDPRVAYWE PAKWVSKLRECKTDNNE ILLNIDMESGHFSAKDRYKFWKESAIQQAFVCK 719 La LHDPRVAYWE PAKWVSKLREHKTDCNE ILLNMDME SGHFSARDRYKFWKE SAVQQAFVCK 719 Lb FHDPRVAYWE PAKWVTKLREYKTDNNE ILLNMDMESGHFSAKDRYKFWKESAIQQAFVCK 719 EC LHDSQVQYWE PAKWVAKLRELKTDDHLLLLCTDMDSGHGGKSGRFKSYEGVAMEYAFLVA 674 Se LHDSQVQYWE PAKWVAKLRELKTDQRLLLLCTDMDSGHGGKSGRFKSYEGVALEFAFLIG 674

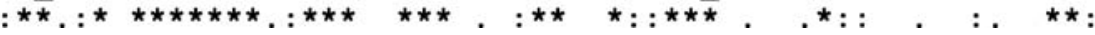

The analysis of Leishmania oligopeptidase B gene expression was performed using total RNA prepared from each stage and detected with specific oligonucleotide primers. The oligopeptidase B gene and oligopeptidase B activity were observed during all life cycle stages of $T$. cruzi (Burleigh et al. 1997). As observed in T. cruzi, results indicated that the oligopeptidase B gene is expressed in promastigotes (logarithm and stationary phases) and axenic amastigotes (Fig. 2), indicating a conservation of gene expression during the entire life cycle in Leishmania and Trypanosoma genus.

Mapping of the catalytic domain, subsites, and oxanion

To date, no oligopeptidase B enzymes have been identified in or cloned from mammalian cells. Oligopeptidase B enzymes have been identified only in prokaryotes and kinetoplastid protozoan parasites. Several studies have biochemically characterized bacterial and trypanosome oligopeptidases B. Using alignment of bacterial oligopeptidases with trypanosomatid oligopeptidases, we determined a catalytic triad, subsites $\mathrm{S} 1$ and $\mathrm{S} 2$, from L. amazonesis (Table 4). We observed conservation in the catalytic triad and S1 subsite; however, a difference was observed in the S2 subsite. Using site-directed mutagenesis and molecular modeling, a pair of residues, Asp 460 and Asp 462, was defined for bacterial oligopeptidase $\mathrm{B}$ that may be involved in defining P2 specificity (Morty et al. 2002; Gerczei et al. 2000). Trypanosome oligopeptidases B demonstrate great similarity, as they have two glutamic acids at the same position (Morty et al. 1999). However, in oligopeptidases from L. amazonensis, L. major, and L. infantum, aspartic 


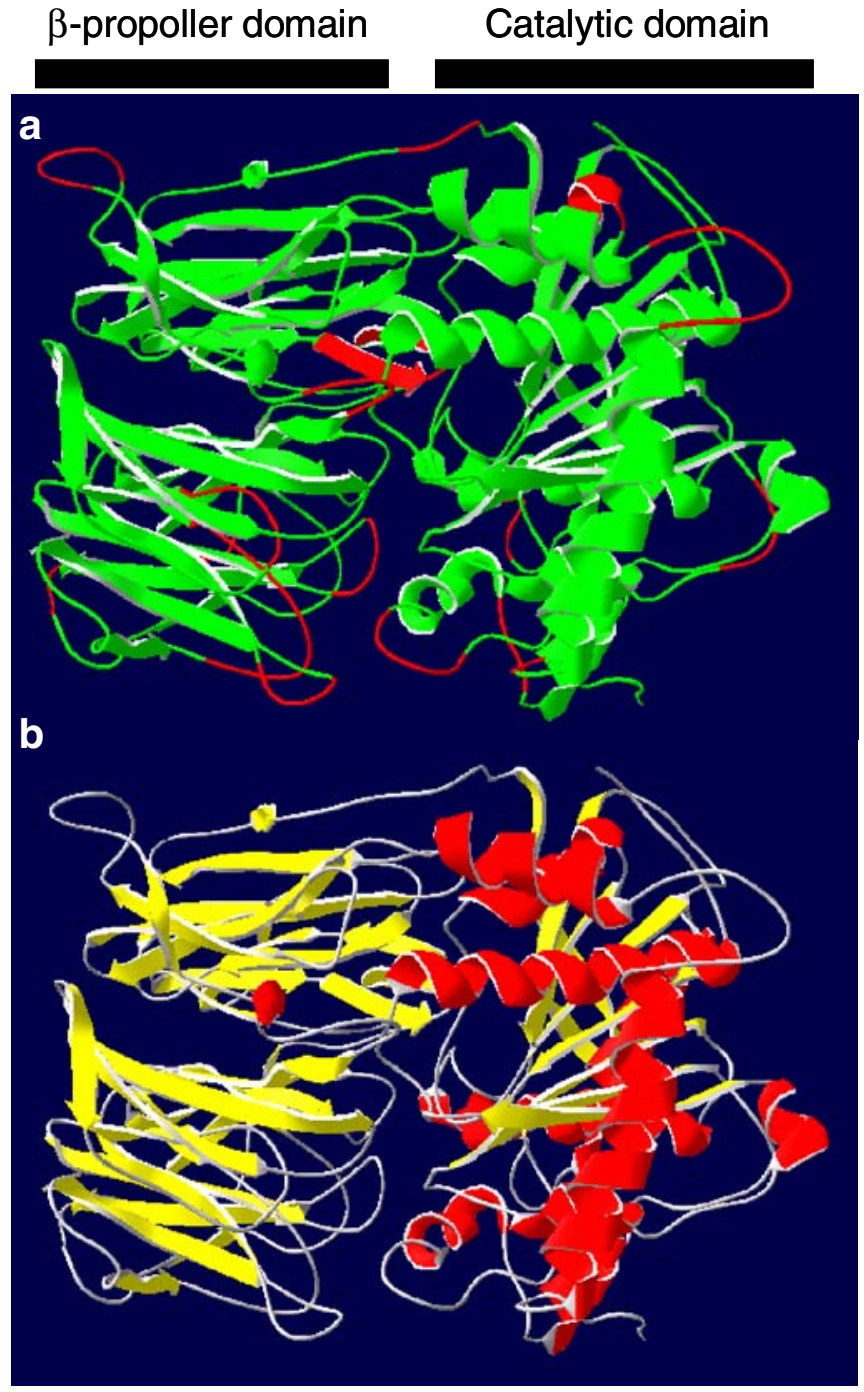

Fig. 5 Molecular modeling of oligopeptidase B from L. amazonensis. a La_OpB structure colored by B-factor. Cold residues are shown in green, while hot residues are in red. b La_OpB molecular model ribbon representation colored for secondary structure (red, $\alpha$ helices;

acid and glutamine were observed at the same position, and in L. braziliensis, aspartic acid and lysine were observed. The substitution of aspartic acid or glutamic acid for glutamine or Lys at the S2 position demonstrates different biochemical characteristics mainly for Lysine. These modifications suggest different substrate specificities based at the P2 position or that other residues may be involved in preferential cleavage by $\mathrm{OpB}$ after the pair of basic residues.

Leishmania ssp oligopeptidases B display Tyr-496 and Ala-533 at the same positions as Tyr-452 Ala 533 oxanion binding sites of oligopeptidase B from Echerichia coli. In trypanosome oligopeptidases B, Tyr and Ala were observed at the same positions, indicating the conservation of an oxanion position in oligopeptidases B. In oligopeptidase B from T. brucei, $\mathrm{C} 256$ was identified as the reactive cysteine

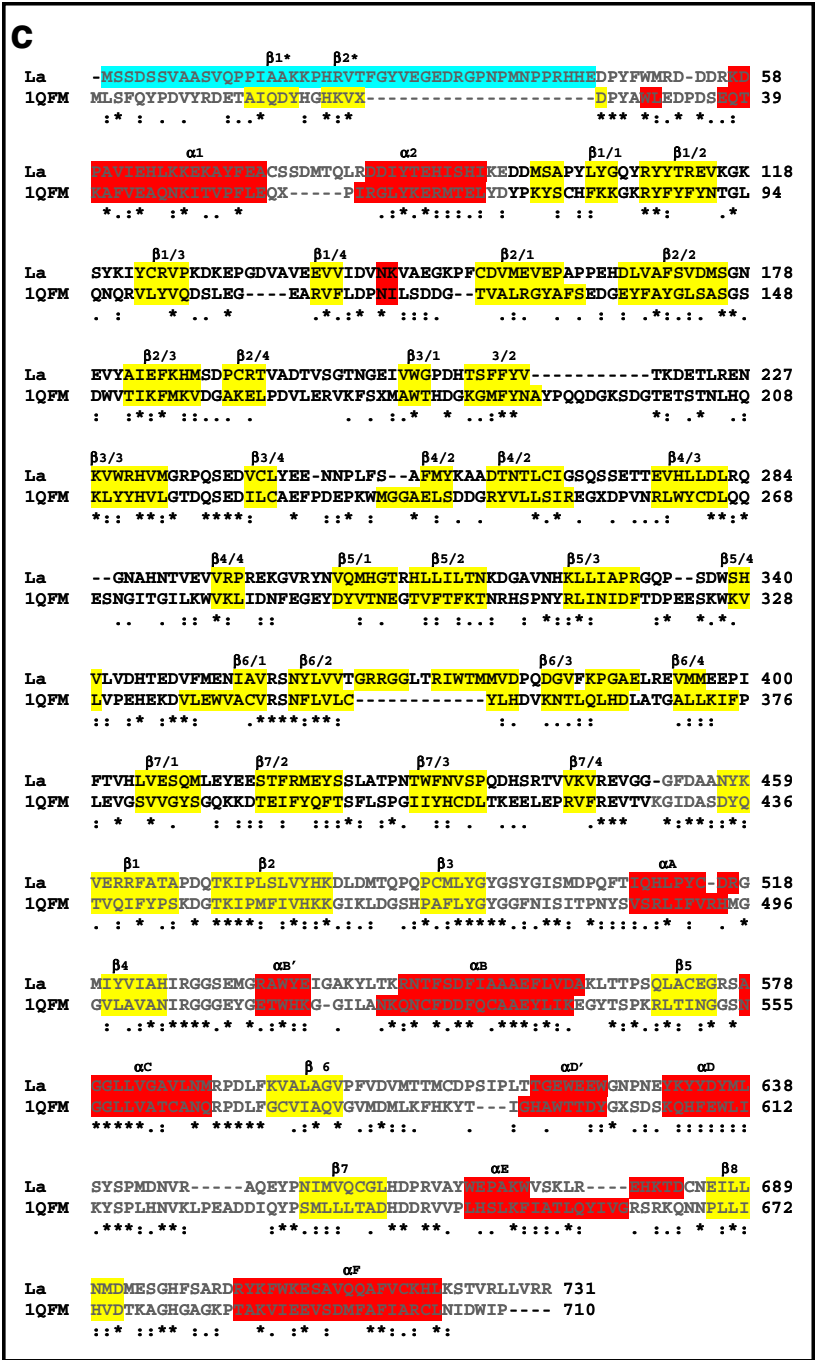

yellow, $\beta$ strands; gray, loops). c Multiple sequence alignment of La_OpB and prolyl oligopeptidase from pig used in the construction of the model. Secondary elements are represented in yellow ( $\beta$ strands) and in red ( $\alpha$ helices)

residue that mediates $\mathrm{OpB}$ inhibition by $N$-ethylmaleimide and iodoacetic acid. Modeling studies suggest that C256 adducts occlude the P1 substrate-binding site, preventing substrate binding (Morty et al. 2001). This cysteine is absent in Leishmanias oligopeptidase B, suggesting the absence of inhibition by $\mathrm{N}$-ethylmaleimide and iodoacetic acid as observed for OpB from T. cruzi.

Molecular modeling of oligopeptidase B

from L. amazonensis

No information regarding the three-dimensional structure by crystallization for oligopeptidase $\mathrm{B}$ is available; however, oligopeptidase B from $E$. coli has been modeled using prolyl oligopeptidase from porcine brain (PEP) as a mold. Using homology modeling, the three-dimensional 
model was constructed for oligopeptidase B of $L$. amazonensis based on the structure of PEP and PEP_Mx. The final sequence alignment between La_OpB and PEP is shown in Fig. 2c. The optimal alignment contains six insertions and seven deletions of La_OpB to PEP. The insertion at position 25 to 44 induces a fatal error in the procedure of the molecular model (Figs. 3 and 4). La_OpB was modeled at position 46 to 731 . The structure of this oligopeptidase B resembles that of prolyl oligopeptidases
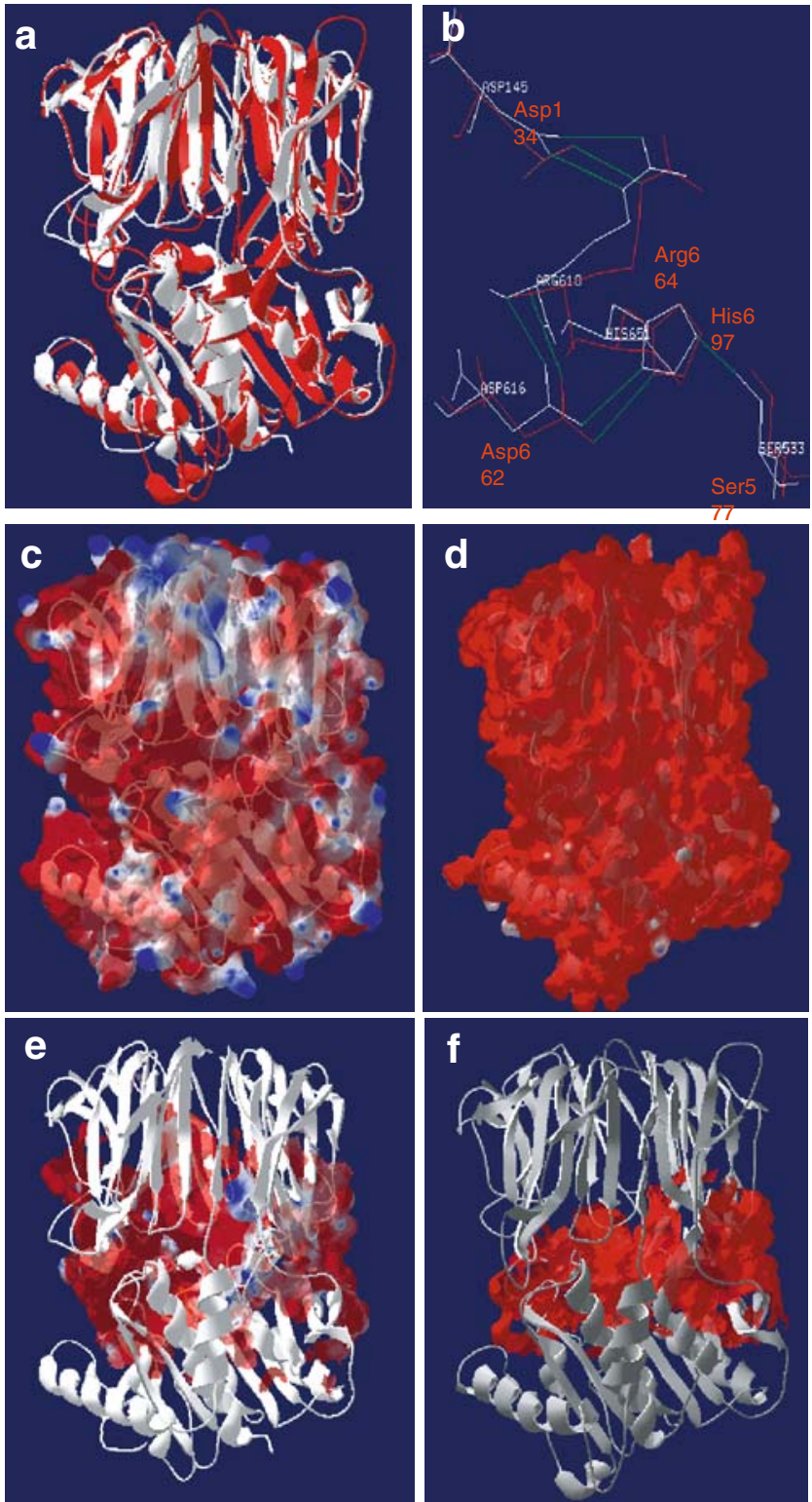

Fig. 6 Superimposition of PEP_Mx on La_OpB. a Superimposition of PEP_Mx (white) and La_OpB (red) using ribbon representation. b The conserved salt bridge between the $\beta$-propoller domain with the catalytic domain present in PEP_Mx (white) and La_OpB (red). c and d The surfaces of PEP Mx and La_OpB were mapped, respectively. $\mathbf{e}$ and $\mathbf{f}$ The surface of the cavity at the interface between the $\beta$ propoller domain and the catalytic domain. The surface was colored according to the Coulombic electrostatic potential: red, negative; gray, neutral; and blue, positive
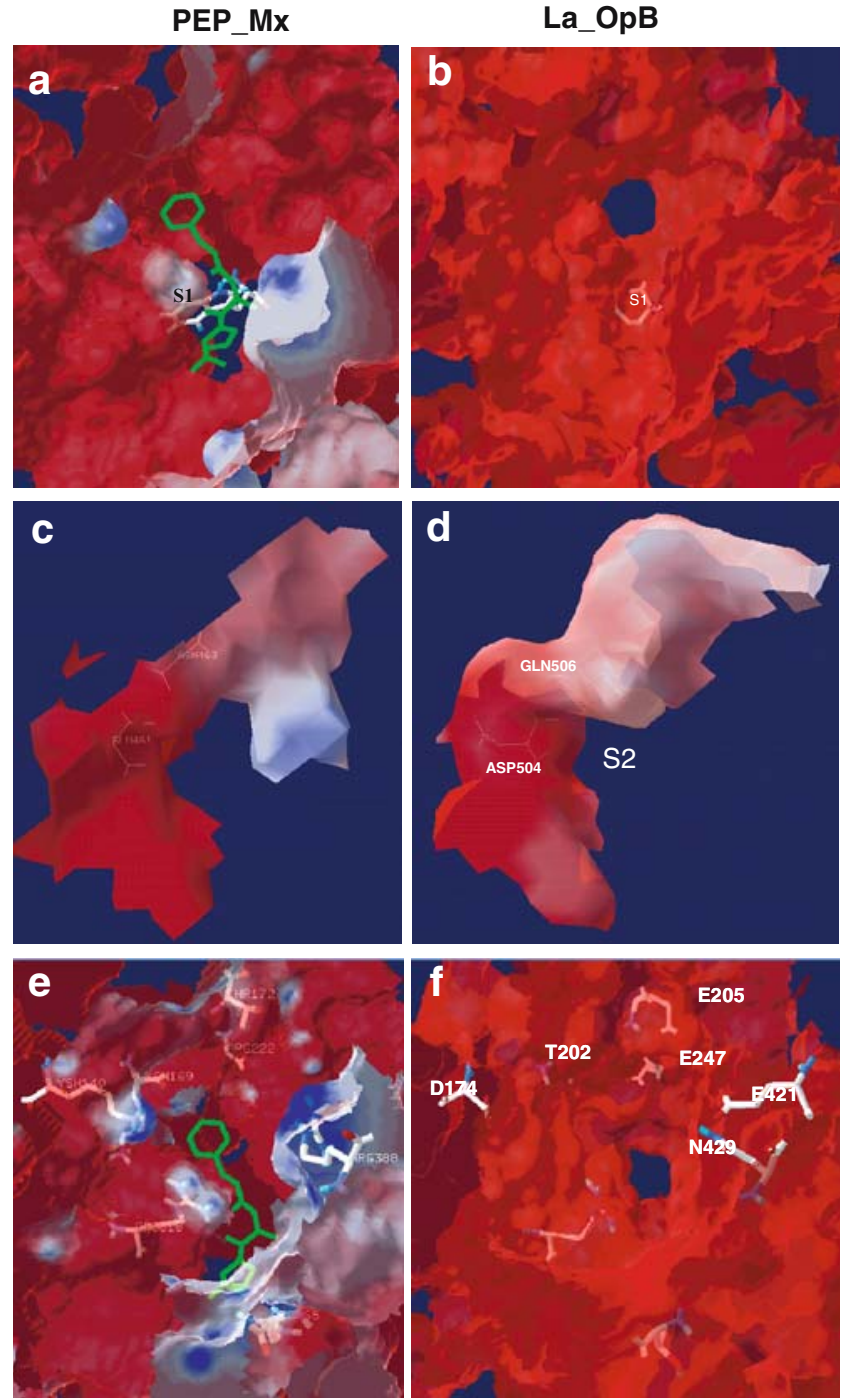

Fig. 7 Electrostatic potential analysis of subsite vicinity of La_OpB. The surface of the cavity was mapped. The surface was colored according to the Coulombic electrostatic potential: red, negative; gray, neutral; and blue, positive. a and $\mathbf{b}$ Top view of S1 subsite of PEP_Mx and La_OpB. c and $\mathbf{d}$ Top view of the S2 subsite of PEP_Mx and La_Op $\bar{B}$. e and $\mathbf{f}$ Comparison of the cavity subsite vicinities of PEP_Mx and La_OpB

(Fig. 5). The insertion at position 365 to 376 promotes the greatest difference in La_OpB and PEP, a $\beta$ strand segment at the interface of the $\mathrm{B}$ propoller domain and catalytic domain. This segment is in the vicinity of the active site in OpB (15 $\AA$ away from the catalytic ser). This structure was absent in the molecular model of oligopeptidase B of E. coli (Gerczei et al. 2000). As this segment is absent in the PEP, the correct folding will be characterized after resolution of the structure of oligopeptidase B from T. brucei (Rea et al. 2006). Furthermore, a helical segment from Gln-587 to Lys-593 has been identified in the molecular model of $\mathrm{OpB} \_$. coli, considering the absence in PEP; however, using our alignment, we observed a 
Table 5 Comparison of residues present in the cavity of prolyl oligopeptidase from $M$. xanthus and oligopeptidase B from $L$. amazonensis

\begin{tabular}{ll}
\hline PEP_Mx & La_OPB \\
\hline K140 & D 174 \\
K169 & T 202 \\
T172 & E 205 \\
R 222 & E 247 \\
V 380 & E 421 \\
R 388 & N 429 \\
R 465 & T 508 \\
\hline
\end{tabular}

corresponding helical segment from Tyr-631 to Leu-638 that is present in PEP at the Lys-605 to Ile-612 position. The other deletions and insertions may be considered as non-breaking gaps. The differences observed between the molecular model of PEP and La_Opb demonstrate low identity/similarity between them and compared to PEP, which induced differences in alignment.

Characterization of the subsite vicinity of oligopeptidase B from L. amazonensis

The preference of oligopeptidase B for cleavage after paired basic residues is intriguing, as these sites are abundant in precursors of biologically active molecules and are recognized as sites for processing and are based on S1 and S2 subsites recognition. To investigate the differences observed in subsite S2, we superimposed the structure of La_OpB on the prolyl oligopeptidase of $M$. xanthus (PEP_Mx; Fig. 6a), resulting in a good fit as expected. We observed conservation in the salt bridge between catalytic and $\beta$ propoller domains observed in the PEP_Mx in La OpB, Asp-134 of $\beta$-propoller and Arg-664 of the catalytic domain (Fig. 6b). The surface analysis of La_OpB demonstrated a mainly negative charge (Fig. 6d) in contrast to the surface of PEP (Fig. 6c). At the interface between the B-propoller and the catalytic domain of $\mathrm{La}$-OpB and PEP_Mx display, a mainly negative charge was observed (Fig. 6e and f). We also compared regions in the cavity of the PEP_Mx without negative charges, but with negative charges in La_OpB.

The S1 subsite is present at the same position of the prolyl oligopeptidase and oligopetidase B (Gerczei et al. 2000); we also observed a S1 subsite of PEP_Mx with a neutral electrostatic potential and a S1 La_OpB subsite with a negative charge containing two glutamic acids (Glu-621 and Glu-623). The orientation of the ZAH inhibitor and docking studies with PQPQLPYPQPQLP substrate in the PEP Mx demonstrated a different orientation of substrate binding at the S2 position in relation to the oligopeptidase B S2 subsite as observed by docking studies (Gerczei et al.
2000) and site-directed mutagenesis (Morty et al. 2002). Based on sequence similarity and the electrostatic surface potential, the S2 subsite region from oligopeptidase B displays great similarity with PEP_Mx. La_OpB presents Asp-504/Gln-506 and PEP_Mx presents Glu-461/Asn 463 at the same position (Fig. $7 \mathrm{c}$ and d). The presence of Arg465 in addition to Glu-461/Asn 463 in PEP_Mx absence in La_OpB possibly blocks the access of basic amino acids and interaction at this region (Fig. 7c). As the S2 subsite sequence of Leishmania oligopeptidases B are different from bacterial and trypanosomes oligopeptidases, but similar at PEP_Mx, we investigated other regions at the interface with must significant electrostatic potential differences that may be involved in preference for the cleavage of dibasic amino acids. We analyzed neighboring amino acids up to a distance of $10 \AA$ from the catalytic triad, and these results are summarized in Table 5. The side chain of Asp-504 (S2) was at a $3-\AA$ distance from E-621 (S1). The side chains of D-174, E-205, and E-247 were also at a 3-Å distance from E-621 (S1) and from Asp-504 (S2), indicating the importance of these residues in the substrate recognition by charge interaction via the positive charge attraction conferred by Arg. Docking of a representative substrate, PQPQLPYPQPQLP, at the active site of the PEP_Mx indicated that the $\mathrm{N}$ terminal substrate residues interact extensively with the catalytic domain, and the $\mathrm{C}$ terminal residues stretch into the propeller domain. D-174, E-205, and E-247 are located in the $\beta$-propoller domain, indicating a participation of this domain also in the substrate binding of oligopeptidase B. Importantly, we did not discard the participation of Asp-504/Gln-506 as two carboxyl dyads; however, we believe that other residues may be involved in the protease-substrate interaction for the dibasic cleavage preference.

\section{Conclusion}

In the present study, we cloned and sequenced oligopeptidase B from L. amazonensis. This is the first study to detect oligopeptidase B gene expression during all phases of the Leishmania life cycle. We modeled an oligopeptidase B and discussed possible differences in the S2 subsite. We are currently engaged in protein expression studies to further this biochemical characterization.

Acknowledgment This work was financially supported by the Fundação Oswaldo Cruz, Conselho Nacional de Desenvolvimento Científico e Tecnológico (CNPq) and Fundação de Amparo à Pesquisa do Estado do Rio de Janeiro. We thank Dr. Yara M. Traub-Cseko (FIOCRUZ) for fruitful discussions, Dr. Floriano Paes Silva Júnior (FIOCRUZ) for support in the molecular modeling procedure, Dr. Alberto Martín Rivera Dávila for ITS primers donation and Dr. Carlos Roberto Alves (FIOCRUZ) for help in L. amazonensis amastigote 
axenic obtention. We are grateful to Mr. G. A. de Souza and Mr. J. M. Rezende-Neto for technical assistance in Leishmania cultures. We are greatly indebted to the Genomic Plataform-DNA Sequencing (PDTISFiocruz). H. L. de Matos Guedes was DSc fellow from CNPq.

\section{References}

Alexander J, Russell DG (1992) The interaction of Leishmania species with macrophages. Adv Parasitol 31:175-254

Alves CR, Corte-Real S, Bourguignon SC, Chaves CS, Saraiva EM, (2005) Leishmania amazonensis: early proteinase activities during promastigote-amastigote differentiation in vitro. Exp Parasitol 109:38-48

Altschul SF, Madden, TL, Schaffer AA, Zhang J, Zhang Z, Miller W, Lipman DJ (1997) Gapped BLAST and PSI-BLAST: a new generation of protein database search programs. Nucleic Acids Res 25:3389-3402

Ashall F, Harris D, Roberts H, Healy N, Shaw E (1990) Substrate specificity and inhibitor sensitivity of a trypanosomatid alkaline peptidase. Biochim Biophys Acta 1035:293-299

Barral A, Costa JM, Bittencourt AL, Barral-Netto M, Carvalho EM (1995) Polar and subpolar diffuse cutaneous leishmaniasis in Brazil: clinical and immunopathologic aspects. Int J Dermatol 34:474-479

Bendtsen JD, Nielsen H, von Heijne G, Brunak S (2004) Improved prediction of signal peptides:Signalp 3.0. J Mol Biol 340:783-795

Burleigh BA, Woolsey AM (2002) Cell signalling and Trypanosoma cruzi invasion. Cell Microbiol 4:701-711

Burleigh BA, Caler EV, Webster P, Andrews NW (1997) A cytosolic serine endopeptidase from Trypanosoma cruzi is required for the generation of $\mathrm{Ca}^{2+}$ signaling in mammalian cells. J Cell Biol 136:609-620

Caler EV, Avalos SV, Haynes PA, Andrews NW, Burleigh BA (1998) Oligopeptidase B-dependent signaling mediates host cell by Trypanosoma cruzi. EMBO J 17:4975-4986

Cazzulo JJ (2002) Proteinases of Trypanosoma cruzi: patential targets for the chemotherapy of Changas desease. Curr Top Med Chem 2:1261-1271

Claros MG, Vincens P (1996) Computational method to predict mitochondrially imported proteins and their targeting sequences. Eur J Biochem 241:779-786

Colmenares M, Tiemeyer M, Kima P, Mcmahon-Pratt D (2001) Biochemical and biological characterization of the protective Leishmania pifanoi amastigote antigen P-8. Infect Immun 69:6776-6784

Coutinho SG, Pirmez C, Mendonça SCF, Conceição-Silva F, Dórea BC (1987) Pathogenesis and immunopathology of leishmaniasis. Mem Inst Oswaldo Cruz 82:214-228

Croft SL, Coombs GH (2003) Leishmaniasis-current chemotherapy and recent advances in the search for novel drugs. Trends Parasitol 19:502-508

Dávila AMR (2002) Tripanosomose animal na América do Sul: Epizootiologia,Evolução e Tecnologias da Informação. Tese de doutorado. Fiocruz, RJ

de Castro E, Sigrist CJA, Gattiker A, Bulliard V, LangendijkGenevaux PS, Gasteiger E, Bairoch A, Hulo N (2006) ScanProsite: detection of PROSITE signature matches and proRule-associated functional and structural residues in proteins. Nucleic Acids Res 34:w362-w365

Fülöp V, Böcskei Z, Polgár L (1998) Prolyl oligopeptidase: an unusual $\beta$-propeller domain regulates proteolysis. Cell 94:161-170

Gasteiger E, Hoogland C, Gattiker A, Duvaud S, Wilkins MR, Appel RD, Bairoch A (2005) Protein identification and analysis tools on the ExPASy server. In: Walker John M (ed) The proteomics protocols handbook. Humana Press, pp 571-607

Gerczei T, Keseru GM, Naray-Szabo G (2000) Construction of a 3D model of oligopeptidase $\mathrm{B}$, a potential processing enzyme in prokaryotes. J Mol Graph Model 18:7-17

Guedes HLM, Rezende-Neto JM, Abreu MF, de Salles CMC, De Simone SG (2007) Identification of serine proteases from Leishmania braziliensis. Z Naturforsch C (in press)

Guex N, Peitsch MC (1997) SWISS-MODEL and the SwissPdbViewer: an environment for comparative protein modeling. Electrophoresis 18:2714-2723

Hemerly JP, Oliveira V, Del Nery E, Morty RE, Andrews NW, Juliano MA, Juliano L (2003) Subsite specificity (S3, S2, S1', S2' and S3') of oligopeptidase B from Trypanosoma cruzi and Trypanosomabrucei using fluorescent quenched peptides: comparative study and identification of specific carboxypeptidase activity. Biochem J 373:933-939

Higgins D, Thompson J, Gibson T, Thompson JD, Higgins DG, Gibson TJ (1994) CLUSTAL W: improving the sensitivity of progressive multiple sequence alignment through sequence weighting, position-specific gap penalties and weight matrix choice. Nucleic Acids Res 22:4673-4680

Ivens AC, Peacock CS, Worthey EA, Murphy L, Aggarwal G, Berriman M (2005) The genome of the kinetoplastid parasite, Leishmania major. Science 309:436-442

Laskowski RA, MacArthur MW, Moss DS, Thornton JM (1993) PROCHECK: a program to check the stereochemical quality of protein structures. J Appl Crystallogr 26:283-291

Morty RE, Troeberg L, Pike RN, Jones R, Nickel P, Lonsdale-Eccles JD, Coetzer THT (1998) Trypanosome oligopeptidase as a target for the trypanocidal agents pentamidine, diminazene and suramin. FEBS Lett 433:251-256

Morty RE, Lonsdale-Eccles JD, Morehead J, Caler EV, Mentele R, Auerswald EA, Coetzer TH, Andrews NW, Burleigh BA (1999) Oligopeptidase B fromTrypanosoma brucei, a new member of an emerging subgroup of serine oligopeptidases. J Biol Chem 274:23156-26149

Morty RE, Lonsdale-Eccles JD, Mentele R, Auerswald EA, Coetzer TH (2001) Trypanosome-derived oligopeptidase B is released into the plasma of infected rodents, where it persists and retains full catalytic activity. Infect Immun 69:2757-2761

Morty RE, Fulop V, Andrews NW (2002) Substrate recognition properties of oligopeptidase B from Salmonella enterica serovar Typhimurium. J Bacteriol 184:3329-3337

Morty RE, Pelle R, Vadasz I, Uzcanga GL, Seeger W, Bubis J (2005) Oligopeptidase B from Trypanosoma evansi. A parasite peptidase that inactivates atrial natriuretic factor in the bloodstream in infected hosts. J Biol Chem 280:10925-10937

Murray HW, Berman JD, Davies CR, Saravia NG (2005) Advances in leishmaniasis. Lancet 366:1561-1577

Pacaud M, Richaud C (1975) Protease II from Escherichia coli. J Biol Chem 250:7771-7779

Polgar L (1997) A potential processing enzyme in prokaryotes: oligopeptidase B, a new type of serine peptidase. Proteins 28:375-379

Polgar L (2002) The prolyl oligopeptidase family. Cell Mol Life Sci 59(2):349-362

Rawlings ND, Morton FR, Barrett AJ (2006) MEROPS: the peptidase database. Nucleic Acids Res 34:D270-D272

Rea D, Fulop V (2006) Structure-function properties of prolyl oligopeptidase family enzymes. Cell Biochem Biophys 44:349-365

Rea D, Hazell C, Andrews NW, Morty RE, Fulop V (2006) Expression, purification and preliminary crystallographic analysis of oligopeptidase B from Trypanosoma brucei. Acta Crystallogr Sect F Struct Biol Crystallogr Commun 62:808-810

Ready PD, Lainson R, Shaw JJ (1983) Leishmaniasis in Brazil: XX Prevalence of "enzootic rodent leishmaniasis" (Leishmania 
mexicana amazonensis), and apparent absence of "pian bois" (Leishmania braziliensis guyanensis), in plantations of introduced tree species and in other non-climax forests in eastern Amazonia. Trans R Soc Trop Med Hyg 1:775-785

Ribeiro de Andrade A, SantoroMM, Melo NM, Mares-Guia M (1998) Leishmania (Leishmania) amazonensis: purification and enzymatic characterization of a soluble serine oligopeptidase from promastigotes. Exp Parasitol 89:153-160

Roggwiller E, Bétoulle MEM, Blisnick T, Breton CB (1996) A role for erytrocyte band 3 by the parasite gp76 serine protease in the formation of the parasitophorous vacuole during the invasion of erythrocytes by Plasmodium falciparum. Mol Biol Parasitol 82:13-24

Rosenthal PJ (1999) Proteases of protozoan parasites. Adv Parasitol 43:105-159

Sadij M, McKerrow JH (2002) Cysteine proteases of parasitic organisms. Mol Biochem Parasitol 120:1-21

Sambrook J, Russell DW (2001) Molecular cloning: a laboratory manual, 3rd edn. Cold Spring Harbor Laboratory Press, New York
Shan L, Mathews II, Khosla C (2005) Structural and mechanistic analysis of two prolyl endopeptidases: role of interdomain dynamics in catalysis and specificity. Proc Natl Acad Sci USA 102:3599-3604

Silva FP Jr, De-Simone SG (2004) S1 subsite in snake venom thrombin-like enzymes: can S1 subsite lipophilicity be used to sort binding affinities of trypsin-like enzymes to small-molecule inhibitors? Bioorg Med Chem 12:2571-2587

Silva-Lopez RE, De-Simone SG (2004a) Leishmania (Leishmania) amazonensis: purification and characterization of a promastigote serine protease. Exp Parasitol 107:173-182

Silva-Lopez RE, De-Simone SG (2004b) A serine protease from detergent soluble extract of Leishmania (Leishmania) amazonensis. Z Naturforsch 59C:590-598

Silva-Lopez RE, Coelho MGP, De-Simone SG (2005) Purification of an extracellular serine protease from Leishmania (Leishmania) amazonensis. Parasitology 130:1-13

Venalainen JI, Juvonen RO, Mannisto PT (2004) Evolutionary relationships of the prolyl oligopeptidase family enzymes. Eur J Biochem 271(13):2705-2715 